\title{
Determining Boundaries between Abundance Biozones Using Minimal Equipment
}

\section{Brent Wilson, ${ }^{1}$ Richard Dawe, ${ }^{1}$ Anesh Gopee, ${ }^{2}$ Sherezz Grant, ${ }^{1}$ Arianna Kissoon, ${ }^{1}$ Taneja Young, ${ }^{1}$ Chanelle De Noon, ${ }^{1}$ Annalize McLean, ${ }^{1}$ and Kala Singh ${ }^{1}$}

\author{
${ }^{1}$ Petroleum Geoscience Programme, Department of Chemical Engineering, Faculty of Engineering, \\ The University of the West Indies, St. Augustine, Trinidad and Tobago \\ ${ }^{2}$ Department of Land Science and Surveying, Faculty of Engineering, The University of the West Indies, \\ St. Augustine, Trinidad and Tobago
}

Correspondence should be addressed to Brent Wilson, brent.wilson@sta.uwi.edu

Received 26 February 2010; Accepted 12 May 2010

Academic Editor: Mariana Amato Copyright ( $) 2010$ Brent Wilson et al. This is an open access article distributed under the Creative Commons Attribution License,
which permits unrestricted use, distribution, and reproduction in any medium, provided the original work is properly cited.

\begin{abstract}
The areal extent of a biological community is usually determined using statistical techniques that only give reliable results where samples contain similar and high numbers of specimens. This paper presents a simple, inexpensive method for determining the geographical limits of biological communities applicable where adjacent samples contain widely differing numbers of specimens. The method is a development of SHE Analysis, which discerns boundaries between adjacent abundance biozones ( $\mathrm{ABs}$ ), an $\mathrm{AB}$ being an area with a distinct community structure. As originally conceived, $\mathrm{SHE}_{\mathrm{bi}}$ (SHE Analysis for the identification of Biozones) commences with species' absolute abundances and works best with large samples of equal sizes. If the variance in $N$ (per sample) is high, $\mathrm{SHE}_{\mathrm{bi}}$ may place $\mathrm{AB}$ boundaries in unexpected locations. A modification, based on proportional abundances, is developed here using species' proportional abundances $\left(p_{i}=n_{i} / N\right)$ for each sample where $n_{i}$ is the number of specimens in the $i$ th species in the sample. For intertidal foraminifera from the Caroni Swamp, Trinidad, where $N$, the number of specimens, fluctuates widely between samples, the modification $\left(\mathrm{SHE}_{\mathrm{bip}}\right)$ gives ecologically more sensible results than does traditional $\mathrm{SHE}_{\mathrm{bi}}$.
\end{abstract}

\section{Introduction}

“... a statistical analysis or test is not endowed with metaphysical properties; it cannot create good results from bad data!" [20, page 9]

A biological community is a group of interdependent organisms that lives and interacts within a habitat, such as fishes on a coral reef, birds in a forest canopy, or foraminifera within a mangrove swamp. The development of robust quantitative methods for grouping similar samples taken from the same biological community is vital for the recognition of biological communities that are real and not mere statistical artefacts. The boundaries between adjacent biological communities are detected using variations in assemblages of species across an area. The different communities contain either different dominant species or different species altogether. The programmes used to determine these boundaries are usually useful only in limited circumstances where sample sizes are uniform and large. A primary goal of both ecology and paleoecology is to understand the patterns by which groups of species are associated and distributed on the biosphere both at present and through geologic time. This paper presents a novel technique for discerning the boundaries between biological communities that require only Microsoft Excel, or a similar spreadsheet programme, and can be applied to data where the variance in sample size is high.

\section{The Basis of $\mathrm{SHE}_{\mathrm{bi}}$}

SHE Analysis for Biozone Identification (hereafter abbreviated as $\mathrm{SHE}_{\mathrm{bi}}$ ) is a relatively new technique that groups samples within an abundance biozone (AB) by accumulating species' abundance data one sample at a time along a transect [1], an $\mathrm{AB}$ being an area within which the proportional 
abundance of a particular species or group of species differs significantly from that in adjacent areas.

To demonstrate how a $\mathrm{SHE}_{\mathrm{bi}}$ is conducted, this paper first defines some basic terms (see also Glossary of Symbols). It uses as an example the steps followed in this study of intertidal foraminifera in the Caroni Swamp, Trinidad, a small island developing state located in the SE Caribbean Sea.

The intertidal area in this swamp supports a population of foraminifera-the population being the set of all the foraminifera living within the study area. First, a cupful of intertidal sediment is collected from the study area. It is washed to remove clay, silt, and large fragments of organic matter to leave sand and a sample of intertidal foraminifera-the sample being a subset of the population. One specimen - a single foraminiferia-is picked from the sample and identified. A second specimen is then picked and identified. Biological communities usually comprise a number of species. The second specimen could thus belong to either the same species as the first or to a new one. Consequently, the number of species $S$ of foraminifera identified from the sample will increase as the number of specimens $N$ increases. As further foraminifera are picked and identified, a total of $N$ specimens are accumulated from the one sample for which $N=\sum_{i=1}^{i=S} n_{i}$, where $n_{i}$ is the number of specimens in the $i$ th species in the sample. Species richness $S$ has been considered a measure of diversity (e.g., [2]). Unfortunately, when comparing samples of different sizes, the number of species $S$ identified is not a helpful parameter $[3,4]$ because within a sample $S$ is typically proportional to $N$.

A better measure of diversity is Shannon's [5] information function $H$, which is based on proportional rather than absolute abundances. To obtain $H$ for a sample, the proportional abundance $p_{i}$ of the $i$ th species in the sample is first calculated from $p_{i}=n_{i} / N$. $H$ is then defined by $H=$ $-\sum_{i=1}^{i=S} p_{i} * \ln p_{i}$. This term is also known as the ShannonWeiner Index $[6,7]$. Values of $H$ are typically $1.5-3.5$ and rarely $>4.5[8]$; only when $S>10^{5}$ species (which would require an extremely large sample size $N$ ) is $H>5.0$ [9].

Once $H$ has been obtained for the single sample, $e^{H}$ (the exponential of it) can be calculated. Jost [10] termed $e^{H}$ the "number equivalent" or "effective number of elements" of the information function. It tells the absolute number of equally abundant species that would be needed to produce the calculated value of $H$ [11]. Thus, when all species in a sample are equally abundant, $p_{i}$ is constant across all species and $e^{H}=S$. In practice, species vary in abundance within a population, such that some are common and some are rare in any particular sample and $e^{H}<S$. The extent to which a few species dominate the sample (thus decreasing $\left.e^{H}\right)$ or, conversely, the degree to which species abundances are equitably distributed within it (thus increasing $e^{H}$ ), is termed the sample's population structure [1]. The value of $e^{H} / S$ gives a measure of the degree to which one or a few species dominate, and is termed the equitability index $E$ $[12,13]$. This $E=e^{H} / S$ index ranges from 0 to 1 , with lower values indicating greater dominance by a few species.
Thus we have SHE: $S$ (species), $H$ (information function), and $E$ (equitability index). Figure 1 gives a cartoon of the entire process outlined above.

Taking the natural logarithm of the equitability index, we get $\ln E=H-\ln S$. This shows that $\ln E$ (which will be negative because $0<E<1$ ) is the residual remaining when $\ln S$ is subtracted from $H$. Sheldon [14] has shown that for any one sample, $E$ is dependent on the number of species $S$ and that for any one sample $E$ becomes progressively smaller as $N$ (and $S$ ) increase. It follows that, as $\ln N$ increases, the increase in $\ln S$ must be balanced by changes in either $H, \ln E$ or both. Buzas and Hayek [15] outline possible behaviours of $H$ and $\ln E$.

If a graph of $S$ against $N$ is plotted, within a single sample the relationship between increasing $S$ and $N$ is usually so strong that under ideal circumstances the plot is asymptotic (e.g., [16]). Thus, a plot of $\ln S$ against $\ln N$ forms a straight line $[1,17]$. In actuality, the world is a noisy place and some deviation from a straight line usually occurs. Also, it is frequently necessary to accumulate several hundred specimens before the values of $p_{i}$ become almost constant.

Buzas [18] hypothesised that most populations have a logarithmic series population structure. Hayek and Buzas [1] demonstrated that within a population with a logarithmic series structure, $H$ becomes constant beyond a critical but variable value of $\ln N$ (see also [19]). So if, as an increasing number of specimens $N$ are accumulated, a graph of $H$ versus $\ln N$ is plotted, it will not be horizontal throughout, but will slope upwards until this critical value of $\ln N$ is attained (see [17, Figure 37, Station 1]). Practically, it is found that beyond this critical value, most additional species encountered are usually singletons (i.e., represented by single specimens only). The addition of a singleton to a large sample has negligible impact on $H$, the singleton having a very low proportional abundance [4]. Buzas [18] suggested that the logarithmic series population structure should be used as the null model for determining population structures.

Where a sample is large, usually only an aliquot-a fraction of the total $N$ specimens-is picked. It is nevertheless assumed that these specimens have been taken randomly from an effectively infinite population [20] so that the sample-or an aliquot of it-is statistically representative. Where the population being studied comprises a taxonomically related set of species (e.g., foraminifera) within a community that includes other organisms such as birds, gastropods, and mangrove trees, the taxonomically restricted population (in this case limited to foraminifera) is termed a taxocene [21].

\section{SHE $_{\text {bi }}$}

With the above in mind, $\mathrm{SHE}_{\mathrm{bi}}$ may now be introduced. It is a statistical technique used to identify the point at which the population structure of a taxocene changes as a linear transect of sequential samples crosses a boundary between adjacent abundance biozones (ABs) — that is, crosses the boundary between two areas supporting populations with differing structures (i.e., with species present at differing proportional abundances) or compositions (with new species 


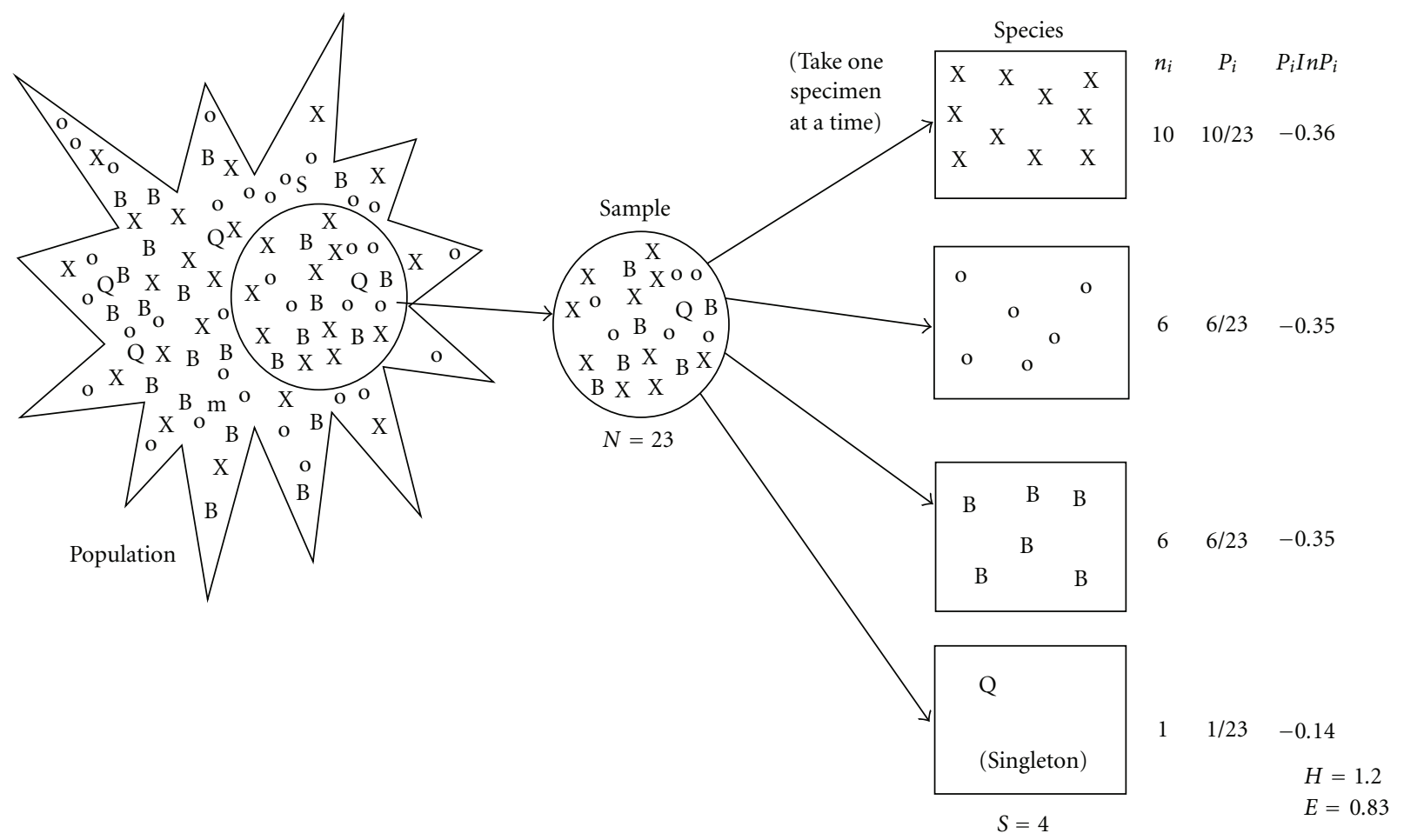

Figure 1: A cartoon showing the procedure in calculating $S, H$, and $E$ for a single sample. Note that $\ln E=H-\ln S$, so in this example $-0.186=1.20-1.386$.

added in significant quantities). SHE $_{\text {bi }}$ can be used to define either modern (ecological-[15]) or ancient (both paleoecological and ecostratigraphic) $\mathrm{AB}$ boundaries based on changes in population structure or composition.

That $\mathrm{SHE}_{\mathrm{bi}}$ is not used widely may in part arise from the tediousness of calculating successive measures using spreadsheets [15]. It may also arise, however, from confusion engendered by a failure by previous workers to distinguish statistical measures obtained from a single sample from those derived from $\geq 2$ accumulated but discrete samples. To overcome this confusion, several symbols are introduced here. $N\left(=\sum_{i=1}^{i=S} n_{i}\right)$ is used to show the number of specimens in a single sample, $L$ to denote the number of samples in the series, and $M$ to indicate the number of specimens in the accumulated samples $L . S_{A}, H_{A}$, and $E_{A}$ are used to distinguish (a) values of these measures as computed from accumulated samples from (b) their values $S, H$ and $E$ as calculated using single samples.

In $\mathrm{SHE}_{\mathrm{bi}}$, samples are accumulated along a line across the study area (a line transect) and $\ln N_{A}, \ln S_{A}, H_{A}$, and $\ln E_{A}$ recalculated as each new sample is added. Buzas and Hayek ([15, Figure 1]) showed using graphs of $\ln S_{A}, H_{A}$ and $\ln E_{A}$ versus $\ln N_{A}$ that all three measures can all change within an area with a uniform population structure (i.e., within an abundance biozone). $H_{A}$ will vary until a sufficient number of specimens have been accumulated to exceed the critical value of $M$ in an area with a logarithmic series population structure. This possibility notwithstanding, $\ln S_{A}, H_{A}$ and $\ln E_{A}$ change more markedly at the point where the line transect moves between $\mathrm{ABs}$ having different population structures. Buzas and Hayek [15] concluded $\ln E_{A}$ versus $\ln N_{A}$ to be the most sensitive indicator of such a transition. The graph of $\ln E_{A}$ versus $\ln N_{A}$ is essentially linear within an $A B$ but shows a marked change in slope at an $A B$ boundary where either (a) sufficient species have joined the accumulated samples to disturb the values of $p_{i}$ for at least some species markedly, (b) species proportions $p_{i}$ within the accumulated assemblage have changed markedly without new species joining the community, or (c) both have occurred.

$\mathrm{SHE}_{\mathrm{bi}}$ uses the successive addition of samples in a series, recalculating the information function $H_{A}$ and related measures (species richness $S_{A}$ and the equitability index $E_{A}$ ) as samples are accumulated. Where an additional sample is the same as the previous samples, there is no significant change in the value of the $H$. This contrasts with raw species richness, which increases with the greater overall sample size and is balanced by a decrease in the equitability index $[7,10,22]$. Crossing an $A B$ boundary results in sampling of a new community, with sharp jumps in $S, H$, and $E$ indicating significant changes in the composition and structure of the population sampled. One challenge facing this cumulative approach is that eventually the accumulating list becomes so large that even the addition of a sample with a substantially different composition needs not have a large effect on $H$ and $E$ [23]. This paper introduces a method termed $\mathrm{SHE}_{\text {bip }}$ for use where the standard deviation of the sample size is high $(>75 \%)$ relative to the mean. 


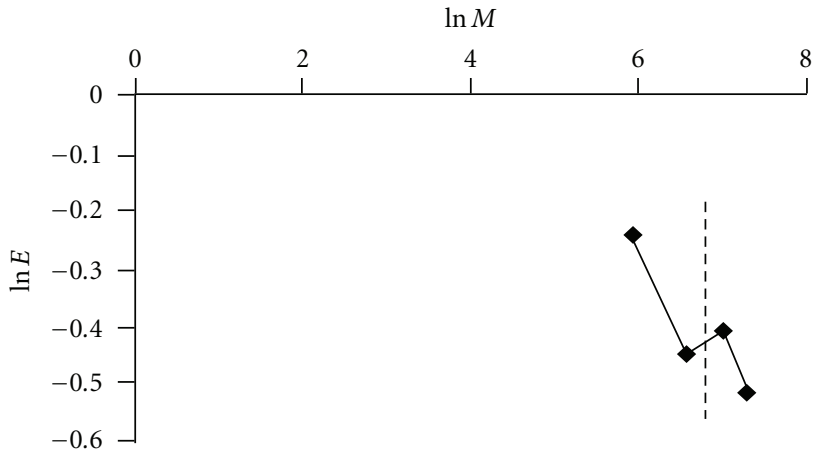

(a)

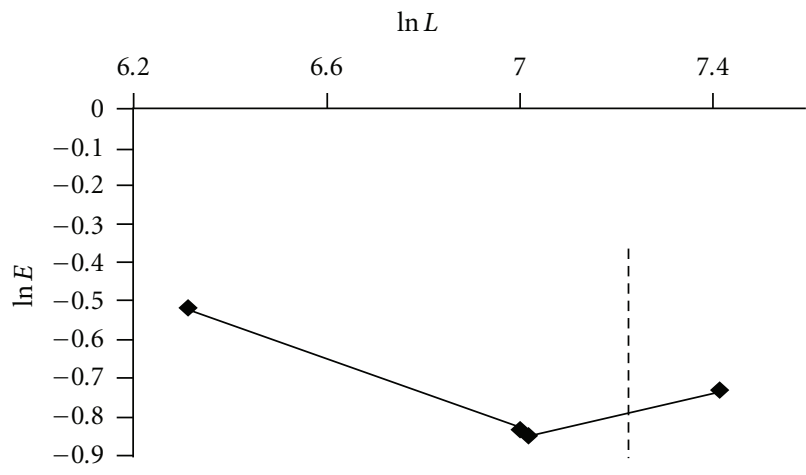

(c)

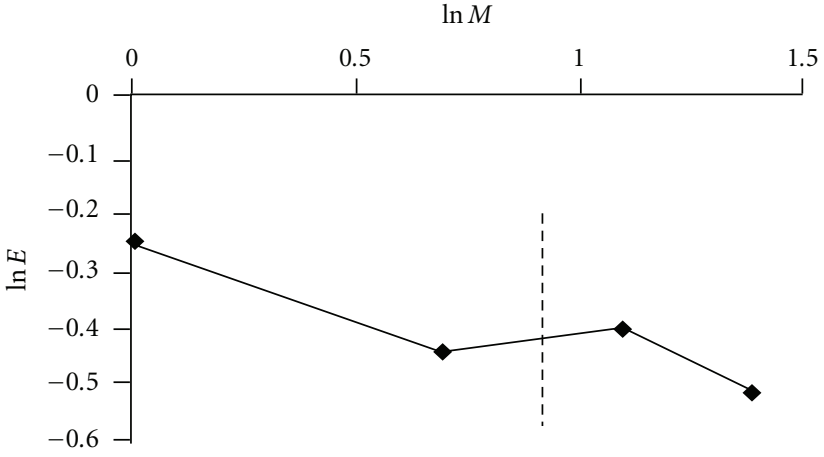

(b)

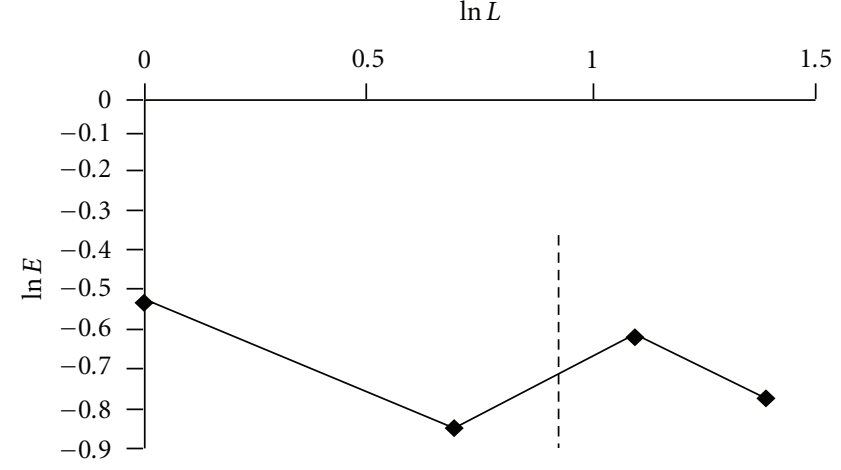

(d)

FIGURE 2: Graphs of $\mathrm{SHE}_{\mathrm{bi}}$ and $\mathrm{SHE}_{\mathrm{bip}}$ analyses of the model data sets. (a) SHE $\mathrm{bi}_{\mathrm{i}}$ applied to Model Data Set 1. (b) SHE bip applied to Model Data Set 1. (c) $\mathrm{SHE}_{\mathrm{bi}}$ applied to Model Data Set 2. (d) $\mathrm{SHE}_{\text {bip }}$ applied to Model Data Set 2.

Under such circumstances, $\mathrm{SHE}_{\text {bip }}$ balances the samples by weighting them all equally, increasing the accuracy with which SHE Analysis identifies ecologically meaningful ABs where $N$ varies widely among samples. $\mathrm{SHE}_{\text {bip }}$ recognizes dissimilar assemblages that are represented by relatively few specimens. Although this phenomenon is investigated using data from intertidal foraminifera from Trinidad, West Indies, it is applicable to all communities.

\section{The Proposed Development: $\mathrm{SHE}_{\mathrm{bi}}$}

$\mathrm{SHE}_{\mathrm{bi}}$ commences with a table of samples and species' absolute abundances such that $M$ comprises the cumulative number of specimens encountered as the samples $L$ are accumulated. In $\mathrm{SHE}_{\text {bip }}$ (SHE Analysis for Biozone Identification based on Proportional Abundances), analysis instead commences with a table of proportional abundance $\left(p_{i}\right)$ data. Since for a single sample $\sum_{i=1}^{i=S} p_{i}=1, S, H$ and $E$ do not differ for that sample whether $\mathrm{SHE}_{\mathrm{bi}}$ or $\mathrm{SHE}_{\text {bip }}$ is used. When a table of proportional abundances is used for $\mathrm{SHE}_{\mathrm{bip}}$, however, $L$ becomes $1,2,3, \ldots, x$, where $x$ is the total number of samples accumulated. Thus, for $\mathrm{SHE}_{\text {bip }}$,

$$
H_{A}=-\sum_{i=1}^{i=L}\left[\left(\sum_{i=1}^{i=S} \frac{p_{i}}{L}\right) \cdots \ln \left(\sum_{i=1}^{i=S} \frac{p_{i}}{L}\right)\right],
$$

where $\sum p_{i}$ is the sum of the proportional abundances of the $i$ th species across all the samples accumulated, and $L$ is the number of samples accumulated. As successive samples are accumulated, $H_{A}$ is recalculated using each species' mean proportional abundances in those samples. Where $N$ varies widely from sample to sample, this will induce differences in $H_{A}$ as compared with $H_{A}$ computed using $\mathrm{SHE}_{\mathrm{bi}}$ (which uses raw abundance data). Nevertheless, because $\ln S_{A}$ is the same for both methods, the relation $\ln S_{A}=H_{A}+E_{A}$ holds true whether $\mathrm{SHE}_{\mathrm{bi}}$ or $\mathrm{SHE}_{\text {bip }}$ is employed and it follows that any differences in $H_{A}$ between $\mathrm{SHE}_{\text {bi }}$ and $\mathrm{SHE}_{\text {bip }}$ must be matched by differences in $\ln E_{A}$. Whereas in $S_{H} E_{b i}$ an $\mathrm{AB}$ boundary is drawn wherever a graph of $\ln E_{A}$ versus $\ln N_{A}$ shows a break in slope, in $\mathrm{SHE}_{\text {bip }}$ it is drawn where there is a break in slope on a graph of $\ln E_{A}$ versus $\ln N_{S}$.

The difference is illustrated here using two model data sets (Table 1, Figure 2) that show how the calculations are made. We used Microsoft Excel for our calculations. In Data Set $1, N$ is constant at 375 specimens per sample and $M$ across all four samples is 1500 . The addition of abundant Species E in sample S3 marks the move from one $\mathrm{AB}$ to another. This is reflected by a change in slope (here an increase) in the graph of $\ln E_{A}$ versus $\ln N_{A}$, no matter whether $\ln E_{A}$ is calculated using $\mathrm{SHE}_{\mathrm{bi}}$ or $\mathrm{SHE}_{\text {bip }}$ (Figures 2(a) and 2(b), resp.). This will not be the case, however, where there are insufficient specimens in the added sample 
TABLE 1: Two model data sets illustrating $\mathrm{SHE}_{\mathrm{bi}}$ versus $\mathrm{SHE}_{\mathrm{bip}}$. (a) Data set in which $N=350$ for all samples. (b) Data set in which $N=550$ for all samples except 33 , in which $N=25$.

(a) Model Data Set 1 .

\begin{tabular}{lcccc}
\hline species & S1 & S2 & S3 & \\
\hline A & 100 & 120 & 50 & 30 \\
B & 120 & 130 & 130 & 135 \\
C & 150 & 120 & 40 & 20 \\
D & 5 & 0 & 5 & 5 \\
E & 0 & 5 & 149 & 1 \\
F & 0 & 0 & 0 & 4 \\
G & 0 & 0 & 375 & 1 \\
\hline$N$ & 375 & 375 & 375 \\
\hline
\end{tabular}

(b) Model Data Set 2 .

\begin{tabular}{lcccc}
\hline Species & $s 1$ & $s 2$ & $s 3$ & \\
\hline$a$ & 350 & 400 & 1 & 1 \\
$b$ & 150 & 140 & 1 & 55 \\
$c$ & 49 & 9 & 0 & 125 \\
$d$ & 1 & 0 & 0 & 0 \\
$e$ & 0 & 1 & 22 & 0 \\
$f$ & 0 & 0 & 0 & 350 \\
$g$ & 0 & 0 & 25 & 1 \\
\hline$N$ & 550 & 550 & 550 \\
\hline
\end{tabular}

S3. In Data Set 2, sample S3 yielded only $N=25$ specimens but marked the first proportionally abundant occurrence of Species F. When examined using $\mathrm{SHE}_{\mathrm{bi}}$ (Figure 2(c)), there is only a slight step between samples S2 and S3 that may be dismissed as being too subtle to be significant (cf. [15, page 237]). The significance of this break can be tested using simultaneous confidence intervals [24], but this can be tedious where a large number of species are involved, simultaneous confidence intervals having to be calculated for every species. Re-examination with $\mathrm{SHE}_{\text {bip }}$ instead reveals a marked step between S2 and S3 indicative of an AB boundary (Figure 2(d)).

\section{Materials and Methods}

Wilson et al. [25] provide a description of the study area, which lies near the mouth of the Blue River in Caroni Mangrove Swamp, Trinidad. Samples of $75 \mathrm{~mL}$ each were taken along three line transects (Figure 3), each sample comprising the top centimetre of sediment. Samples from transects T1 and T2 were taken at $1 \mathrm{~m}$ horizontal intervals, while from the less steeply shelving transect T3 they were collected at $2 \mathrm{~m}$ horizontal intervals. Sample altitudes relative to annual mean sea level (AMSL) were determined using levelling and GPS. Transect T1 lay $\sim 1 \mathrm{~m}$ south of transect C1A of Wilson et al. [25]. Within 48 hours of collection all samples were washed and sieved over a $1 \mathrm{~mm}$ mesh to remove coarse organic fragments, and a $63 \mu \mathrm{m}$ mesh to remove mud and silt. Because this study examined total (live + dead) foraminiferal assemblages, the washed sample residues were stored in fresh water but not stained with rose Bengal.
Foraminifera were picked from the wet residues. An attempt was made to pick $\sim 250$ specimens from all residues, but some yielded considerably fewer. Specimens were identified to species level using especially Todd and Bronnimann [26], Saunders [27, 28], and Boltovskoy and Hincapié de Martínez [29]. Wilson et al. [25] gave brief taxonomic details.

The aim of this paper being to compare how $\mathrm{SHE}_{\mathrm{bi}}$ and $\mathrm{SHE}_{\text {bip }}$ behave where $N$ varies markedly between samples, and not to document how $\mathrm{AB}$ boundaries differed between the three line transects, all three were spliced on the basis of increasing altitude relative to AMSL only. (Other splicing methods, such as ordering samples using detrended canonical analysis, might indicate different $\mathrm{AB}$ boundaries.) $\mathrm{SHE}_{\mathrm{bi}}$ and $\mathrm{SHE}_{\mathrm{bip}}$ were conducted for the three spliced transects and the results compared. $\mathrm{ABs}$ discerned by $\mathrm{SHE}_{\mathrm{bi}}$ were distinguished using italicised uppercase letters, and those indicated by $\mathrm{SHE}_{\text {bip }}$ using italicised numerals.

\section{Results}

6.1. General Characteristics of the Fauna. A total of 34 samples were recovered from the three transects and yielded a total of 3638 specimens of benthonic foraminifera in 33 species. The altitudes of the individual samples relative to AMSL ranged from $-1.18 \mathrm{~m}$ to $0.34 \mathrm{~m}$. For the 34 samples, $N$ varied from 0 to 377 foraminifera $($ mean $=107$, standard deviation [S.D.] = 120.7).

Further analyses were, therefore, restricted to those $L=$ 23 samples ( $\sim 68 \%$ of those collected) that yielded $\geq 20$ specimens (Table 2 ) on the grounds that within these samples $H$ 


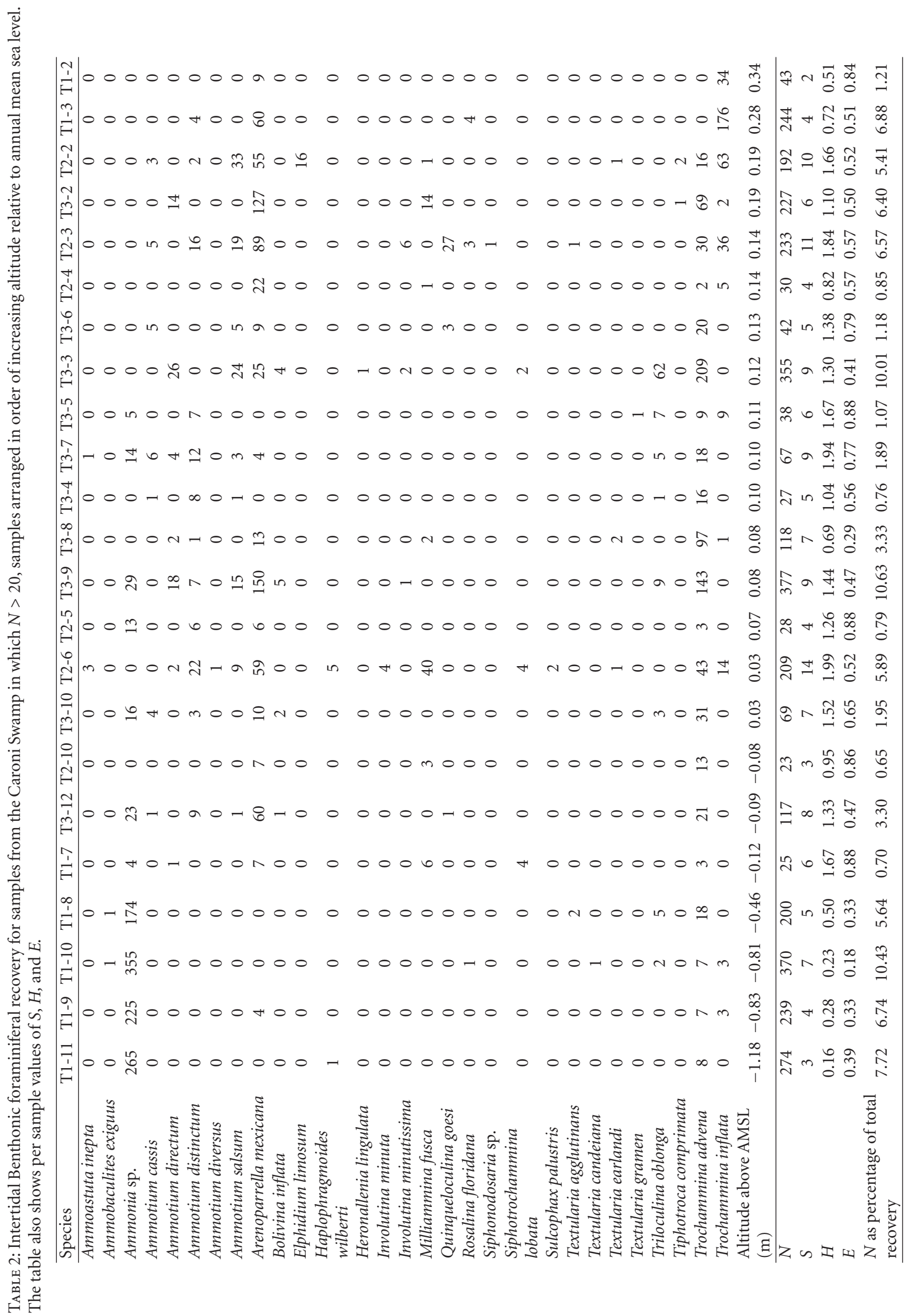



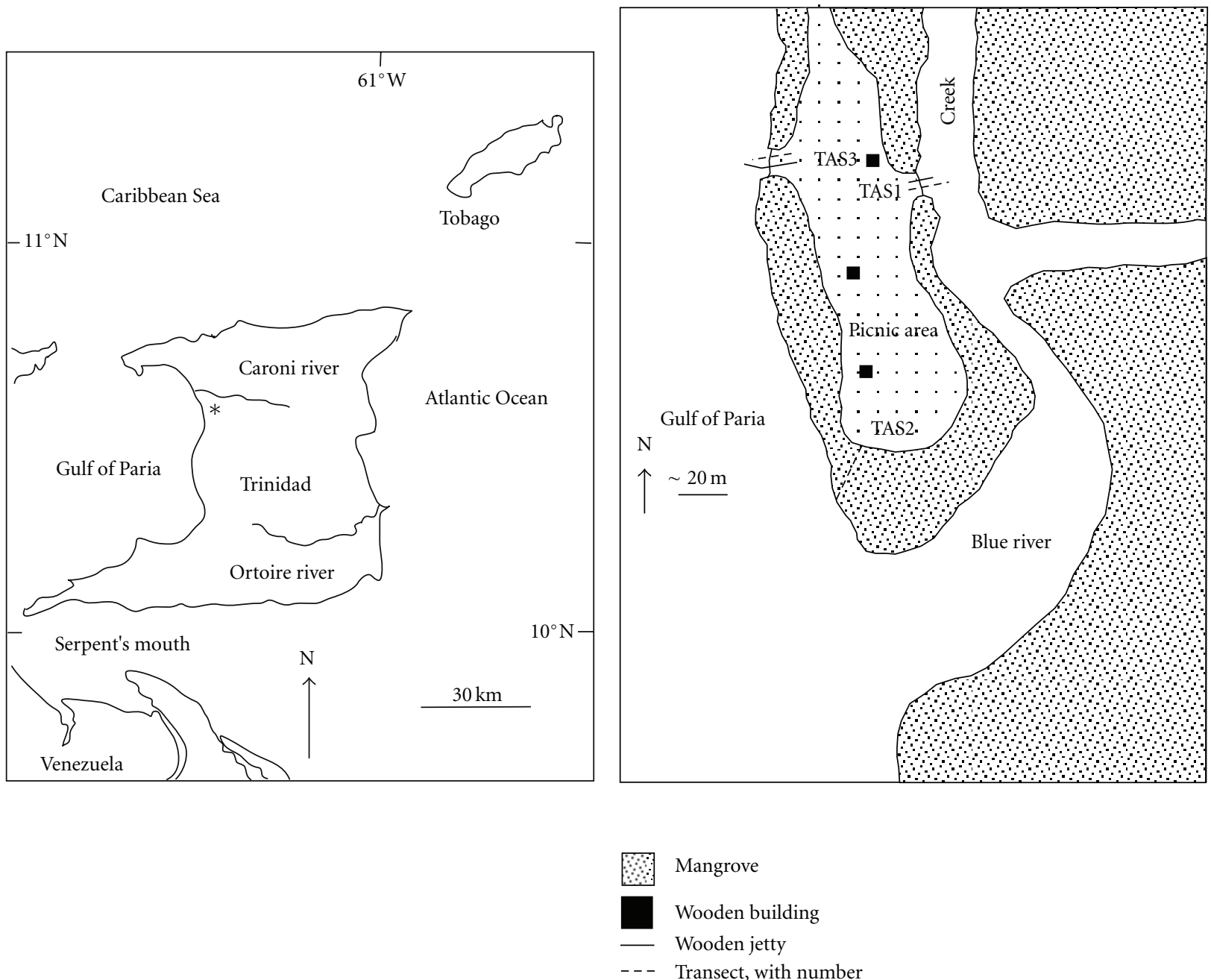

(a)

(b)

FIgURE 3: The location of the study area and sampled transects. (a) Trinidad, showing the location of the Caroni Swamp (asterisk). (b) The area around the mouth of the Blue River, showing the location of the three transects.

was not correlated with $N$. Within these samples the total number of specimens recovered $(M)$ was 3547 foraminifera. The most abundant species were Ammonia sp. (31\% of total recovery from these 23 samples), Arenoparrella mexicana (20\%), Trochammina advena (22\%), and T. inflata (10\%). Ammonia sp. dominated the four samples farthest below AMSL (T1-11 through T1-8), which collectively yielded $\sim 30 \%$ of the total specimens recovered from the 23 samples analysed.

Transect T1 contained 7 of the 23 samples (mean $N=$ 199 foraminifera, S.D. $=125$ ), while T2 contained 6 (mean $N=119$ foraminifera; S.D. $=101)$. Transect T3 contained 10 (mean $N=144$ foraminifera, S.D. $=131$ ). Three samples (T1-10, T3-9 and T3-3, Figure 4) each yielded $>10 \%$ of the total recovery from the 23 samples, and were spread throughout the transects. Five samples yielded $<1 \%$ of the total recovery. Ammonia sp. was most abundant towards the base of the combined transects, Trochammina advena towards the middle, and T. inflata towards the top (Figure 5).

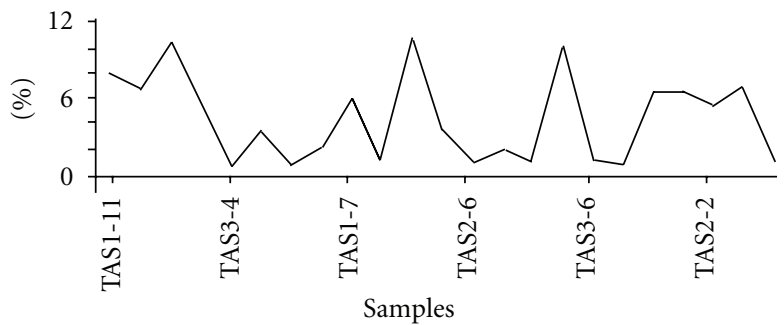

FIGURE 4: Per sample recovery of foraminifera as a percentage of total recovery across all samples in which $N>20$.

Arenoparrella mexicana showed two peaks in proportional abundance.

There was no significant difference between the mean yields of samples from transect T1, with the highest mean, and T2, with the lowest (Student's $t$-test; $t_{\mathrm{obs}}=1.255, t_{\text {crit }}$ $=2.201$, d.f. $=11$ ). Thus, the observed variations in $N$ have 


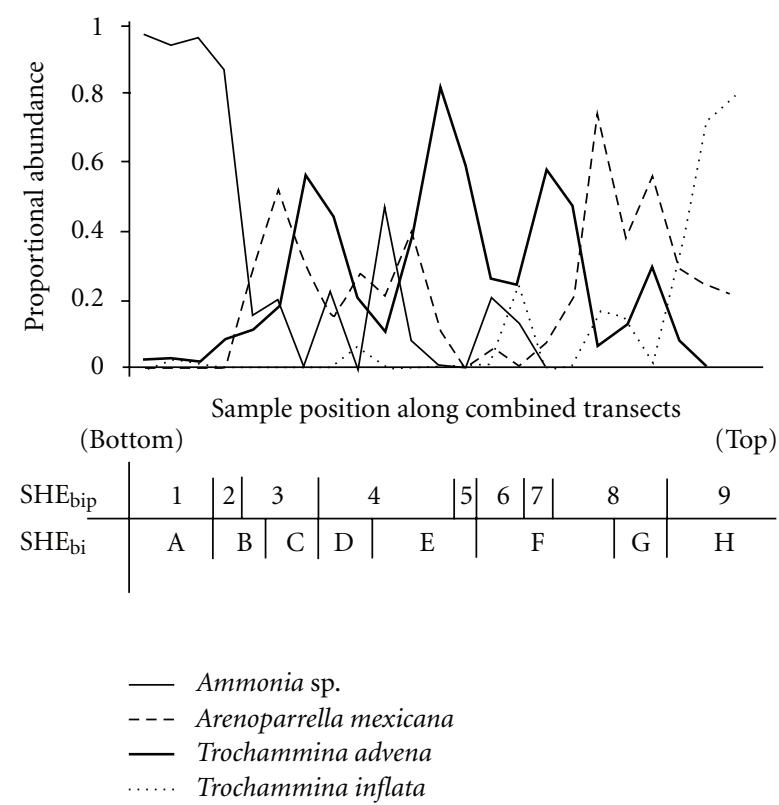

Figure 5: The distribution of the most abundant species across the three transects, and showing the locations of the abundance biozone boundaries as revealed by $\mathrm{SHE}_{\mathrm{bi}}$ and $\mathrm{SHE}_{\mathrm{bip}}$.

not arisen from amalgamating transects with differing mean population densities. For all 23 samples, the mean $N$ was 154 and S.D. 121, the S.D. being $\sim 78 \%$ of the mean. $N$ was insignificantly correlated with $S(r=0.365, P=.087)$ and $H$ $(r=0.237, P=.277)$ but significantly correlated with $E(r$ $=-0.755, P=.001)$. $S$ did not show any trend throughout these samples, but $H$ and $E$ were markedly lower in those four samples near the base of the merged transects that were dominated by Ammonia sp. (Figure 3). For the 23 samples, per sample $N$ as a percentage of the total recovery (i.e., total $M$ ) varied between $1 \%$ and $11 \%$ (mean $4.4 \%$, S.D. $3.4 \%$; Figure 5).

\section{SHE $E_{b i}$ and $S_{H E}$}

Both $\mathrm{SHE}_{\mathrm{bi}}$ and $\mathrm{SHE}_{\mathrm{bip}}$ were applied to the 23 samples with $\geq 20$ specimens. They indicated complex but markedly different patterns of abundance biozones $(\mathrm{ABs}), \mathrm{SHE}_{\mathrm{bi}}$ suggesting there were eight $\mathrm{ABs}$ and nine $\mathrm{SHE}_{\text {bip }}$ (Table 3, Figures 6 and 7). The number of samples per $A B$ as indicated by $\mathrm{SHE}_{\mathrm{bi}}$ ranged from two to five, whereas from $\mathrm{SHE}_{\text {bip }}$ the number ranged from one to five. Sample T3-9, although comprising $>10 \%$ of the recovery, was not differentiated as a separate $\mathrm{AB}$ by either $\mathrm{SHE}_{\mathrm{bi}}$ or $\mathrm{SHE}_{\text {bip }}$. Only four $\mathrm{AB}$ boundaries indicated by $\mathrm{SHE}_{b i}$ coincided with those from $\mathrm{SHE}_{\text {bip }}$, and only two $\mathrm{ABs}$ were identical between the two methods (AB1 from $\mathrm{SHE}_{\text {bip }}$ with $\mathrm{AB} A$ from $\mathrm{SHE}_{\mathrm{bi}}$, and $\mathrm{AB} 9$ from $\mathrm{SHE}_{\text {bip }}$ with $\mathrm{ABH}$ from $\mathrm{SHE}_{\mathrm{bi}}$ ).

\section{Discussion}

The results from both $\mathrm{SHE}_{\mathrm{bi}}$ and $\mathrm{SHE}_{\mathrm{bip}}$ reflect complex fluctuations in the proportional abundances of species
TABLE 3: Comparison of the placing of abundance biozone boundaries when the data from Table 2 are analysed using $\mathrm{SHE}_{\mathrm{bi}}$ versus $\mathrm{SHE}_{\text {bip }}$.

\begin{tabular}{lccc}
\hline Sample & Altitude above AMSL $(\mathrm{m})$ & SHE $_{\mathrm{bi}}$ & SHE $_{\mathrm{bip}}$ \\
\hline TAS1-2 & 0.34 & & \\
TAS1-3 & 0.28 & $H$ & 9 \\
TAS2-2 & 0.19 & & \\
TAS3-2 & 0.19 & $G$ & \\
TAS2-3 & 0.14 & & 8 \\
TAS2-4 & 0.14 & & \\
TAS3-6 & 0.13 & $F$ & 7 \\
TAS3-3 & 0.12 & & \\
TAS3-5 & 0.11 & & 6 \\
TAS3-7 & 0.10 & & \\
TAS3-4 & 0.10 & $E$ & \\
TAS3-8 & 0.08 & & \\
TAS3-9 & 0.08 & & \\
TAS2-5 & 0.07 & & \\
TAS2-6 & 0.03 & & \\
TAS3-10 & 0.03 & & \\
TAS2-10 & -0.08 & & \\
TAS3-12 & -0.09 & & \\
TAS1-7 & -0.12 & & \\
TAS1-8 & -0.46 & & \\
TAS1-10 & -0.81 & & \\
TAS1-9 & -0.83 & & \\
TAS1-11 & -1.18 & & \\
\hline
\end{tabular}

(Figure 5). Examination of the raw data shows, however, that, due to fluctuations in $N$, use of $\mathrm{SHE}_{\mathrm{bi}}$ induced spurious placement of $\mathrm{AB}$ boundaries along the merged transects $\mathrm{T} 1$ through T3.

The lowest four samples (T1-11 through T1-8) yielded 1083 foraminifera ( $\sim 30 \%$ of the total recovery), of which Ammonia sp. per sample ranged from $87 \%$ to $97 \%$ (mean $94 \%)$. In the fourth sample, $N=200$. Arenoparrella mexicana was in these four samples represented by four specimens only (i.e., $\sim 0.4 \%$ of the recovery from them), and Trochammina advena by forty ( $\sim 3.7 \%$ of recovery). Neither Miliammina fusca nor Siphotrochammina lobata were recovered from the lowest four samples. In the succeeding samples T1-7 (for which $N=25$ foraminifera) and T3-12 $(N=117)$, the proportional abundance of Ammonia sp. dropped markedly, comprising only $16 \%$ and $20 \%$ of the recovery from T1-7 and T3-12, respectively. Meanwhile the percentage abundance of A. mexicana increased to $28 \%$ and $51 \%$ of the samples, respectively. Trochammina advena was also more abundant in T1-7 and T3-12 than below, comprising $12 \%$ and $18 \%$ in these two samples, respectively, while $M$. fusca and $S$. lobata formed $24 \%$ and $16 \%$ of the recovery from T1-7, respectively.

The statistical validity of the changes in the proportional abundances of Ammonia sp. and T. advena between T1-8 and T1-7 was tested using simultaneous confidence intervals 

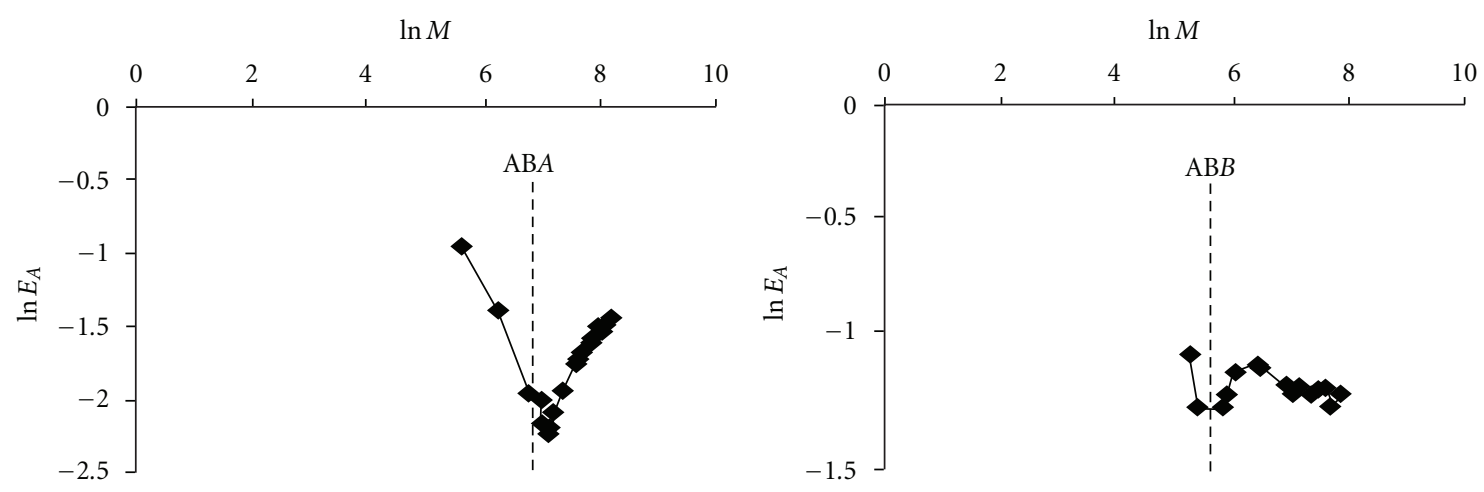

(a)

(b)

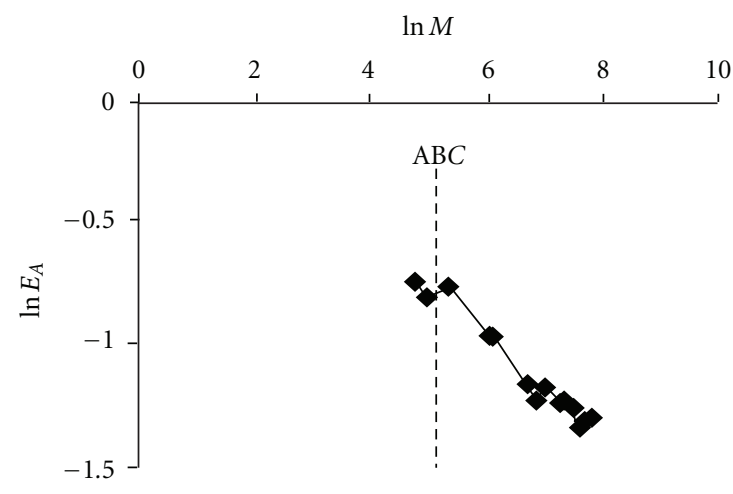

(c)

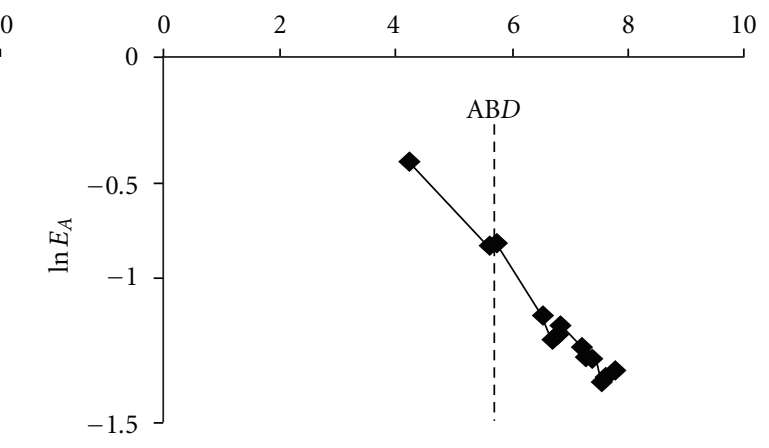

(d)

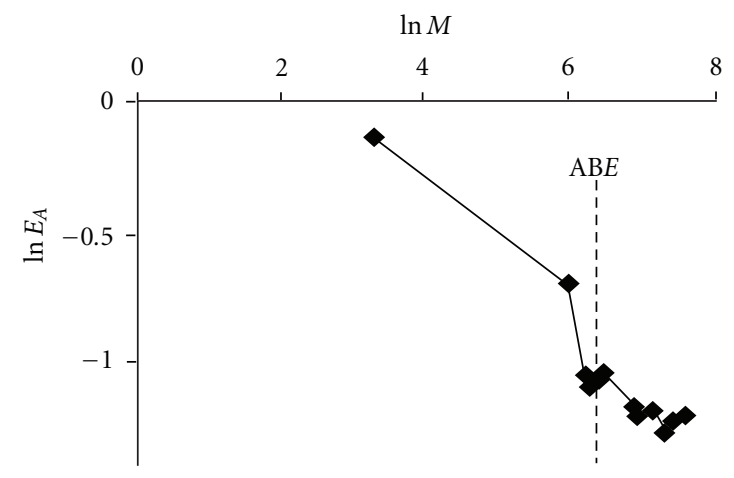

(e)

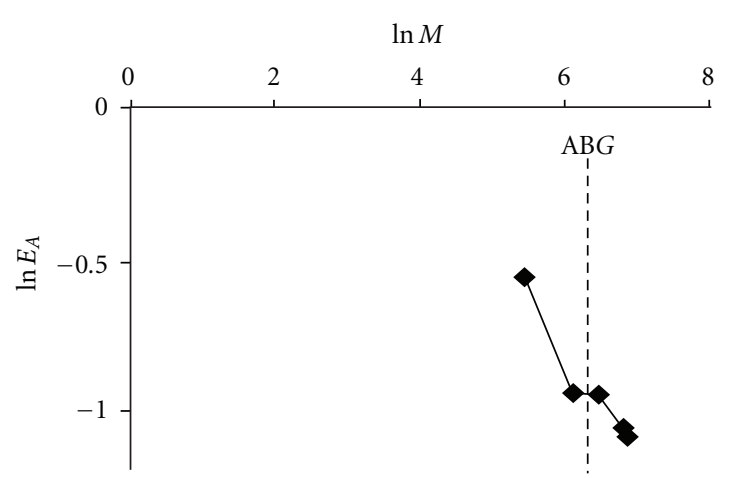

(g)

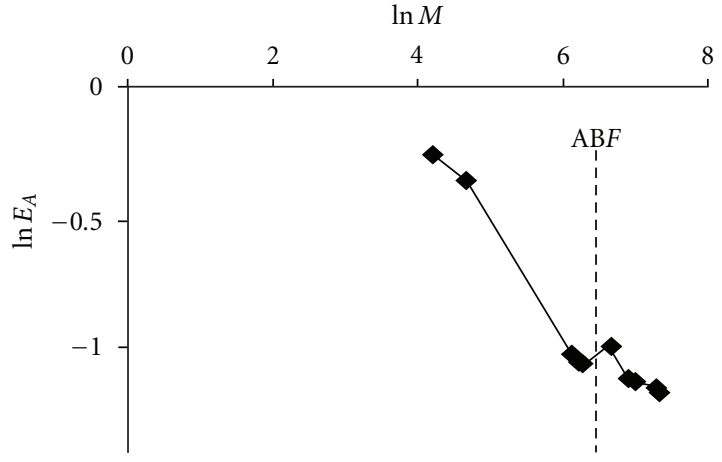

(f)

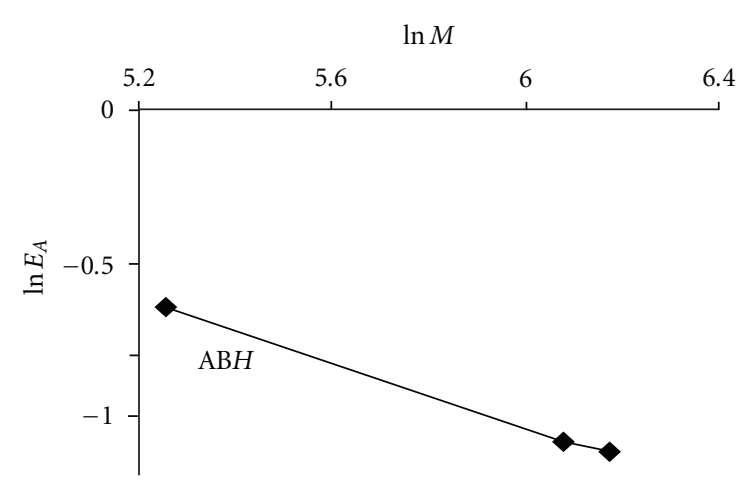

(h)

Figure 6: Plots of $\ln E_{A}$ versus $\ln M$ from $\mathrm{SHE}_{\mathrm{bi}}$ with serial deletions of abundance biozones during the accumulation procedure. Dashed lines indicate the positions at which abundance biozone ( $\mathrm{AB}$ ) boundaries are identified. (a) Grouping of the first 3 samples as $A B A$. (b) Following deletion of the first three samples, grouping of the next two as $A B B$. Figures $6(\mathrm{c})$ through $6(\mathrm{~h})$ continue this sequential procedure until all samples are accounted for. 


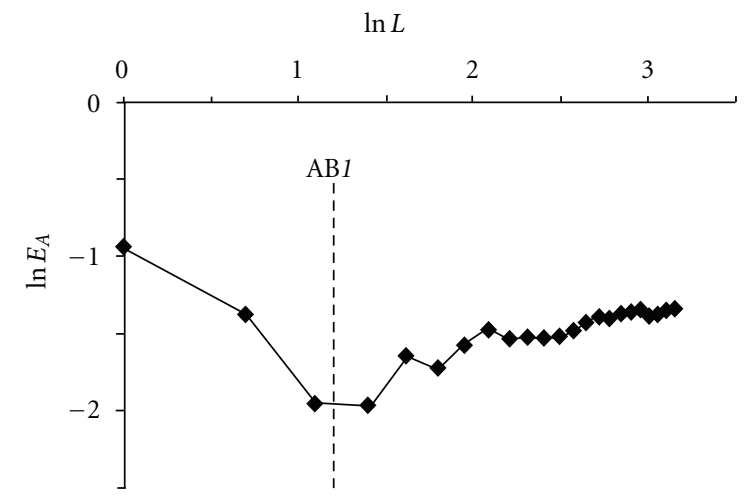

(a)

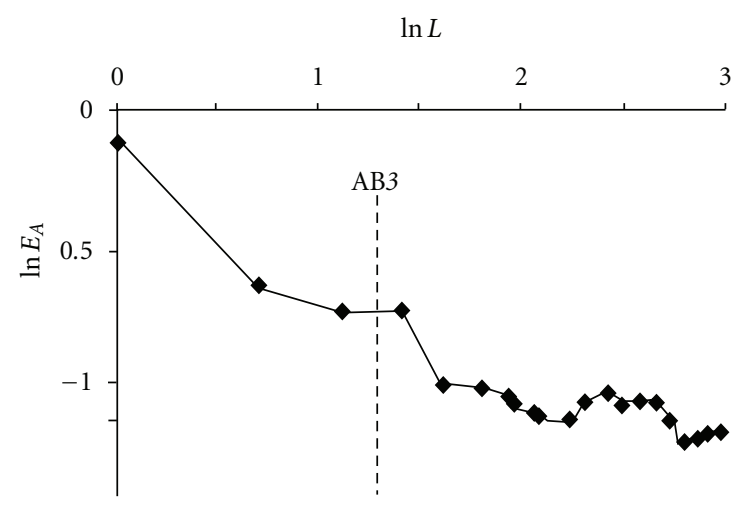

(c)

$\ln L$

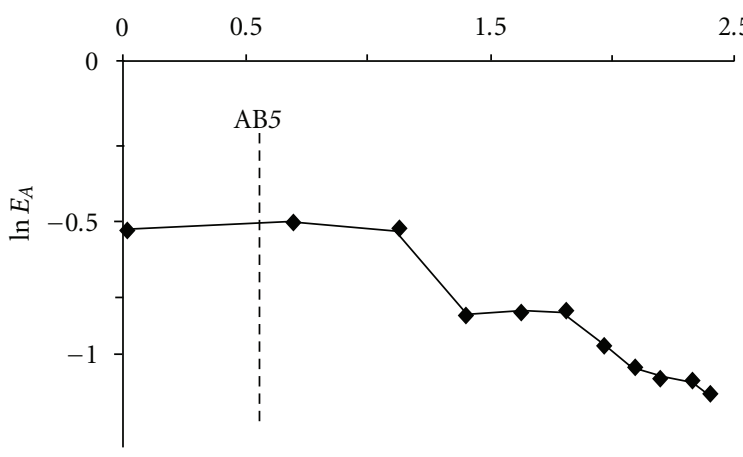

(e)

$\ln L$

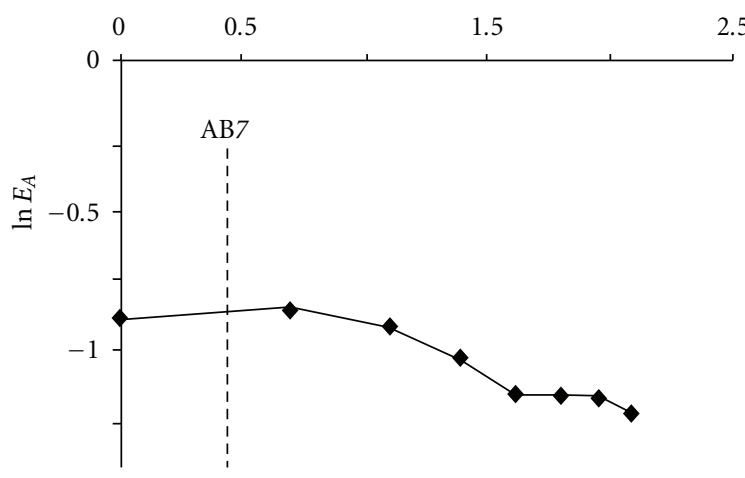

(g)

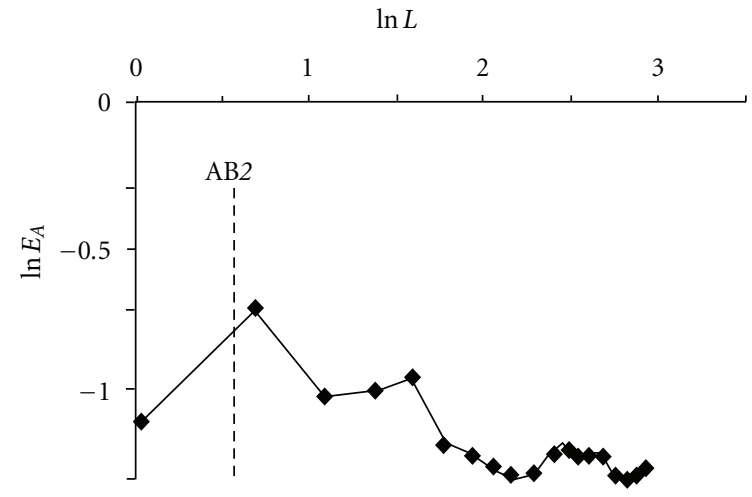

(b)

$\ln L$

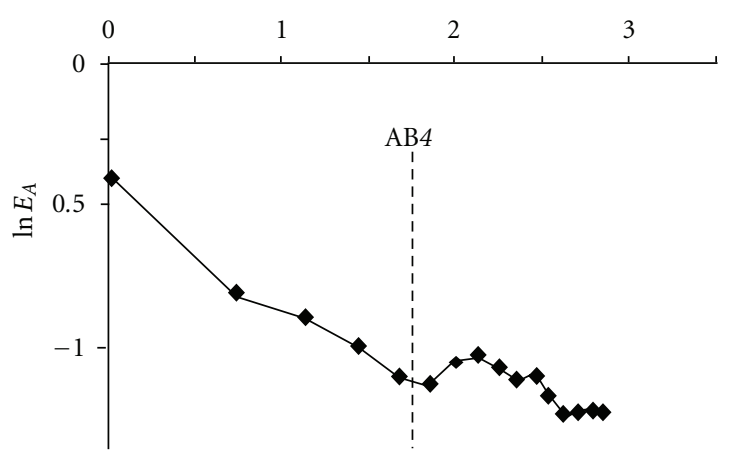

(d)

$\ln L$

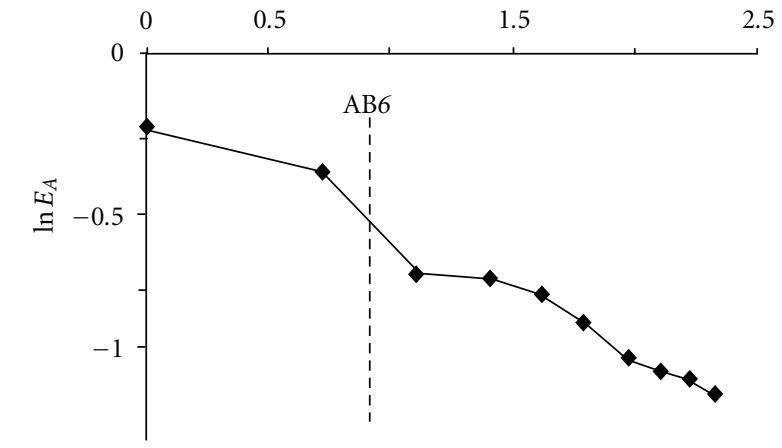

(f)

$\ln L$

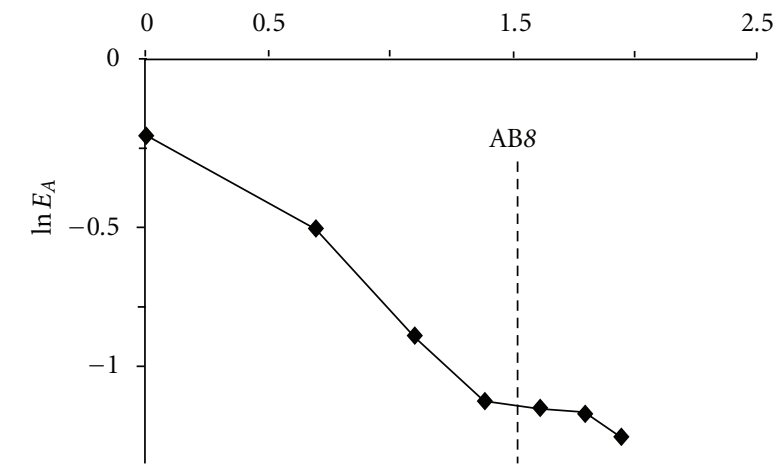

(h)

FIgURE 7: Continued. 


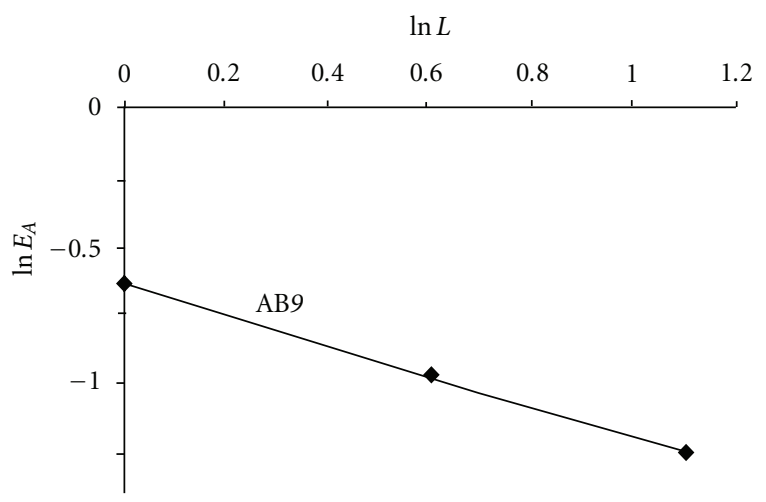

(i)

Figure 7: Plots of $\ln E_{A}$ versus $\ln L$ from $\mathrm{SHE}_{\mathrm{bip}}$ with serial deletions of abundance biozones during the accumulation procedure. Dashed lines indicate the positions at which abundance biozone $(\mathrm{AB})$ boundaries are identified. (a) Grouping of the first 4 samples as $A B 1$. (b) Following deletion of the first four samples, grouping of the next two as AB2. Figures 7(c) through 7(i) continue this sequential procedure until all samples are accounted for.

[24], using a value of $z=2.12$ to avoid a Type II statistical error. (This test could not be applied to A. mexicana, S. lobata and M. Fusca because these were not recovered from T1-8). The results indicate that the decrease in the proportional abundance of Ammonia sp. between T1-8 and T1-7 is statistically significant, but that the change in $T$. advena was not. The decrease in Ammonia sp. being coupled with the appearance of A. mexicana, S. Lobata, and M. fusca, is concluded that there is a change in population structure and composition between samples T1-8 and T1-7.

In line with the above, both $\mathrm{SHE}_{\mathrm{bi}}$ and $\mathrm{SHE}_{\text {bip }}$ placed an $\mathrm{AB}$ boundary after the first three samples. However, whereas $\mathrm{SHE}_{\text {bip }}$ placed a boundary (between ABs 2 and 3) after the first four samples and coincident with the fall in the proportional abundance of Ammonia sp., SHEbi did not, but instead placed the succeeding $A B$ boundary between the fifth (T1-7) and sixth (T3-12) samples. With $\mathrm{SHE}_{\mathrm{bi}}$ it is not until after data from the fifth sample has been accumulated that a sufficient number of specimens of other species have amassed to overpower the high numbers and proportions of Ammonia sp. in the fourth sample. Thus, this difference in boundary placement is due to a coupling of the dominant Ammonia sp. in sample T1-8 with the relatively small $N$ for samples T1-7 and T3-12. Only $\mathrm{SHE}_{\mathrm{bip}}$ was able to overcome the impact of the difference in sample sizes $N$ and delineate an $\mathrm{AB}$ boundary at this point.

In the preceding example, per sample $N$ decreased markedly across the $\mathrm{AB}$ boundary detected using $\mathrm{SHE}_{\mathrm{pa}}$. A second example shows that $\mathrm{SHE}_{\mathrm{bi}}$ may also miss $\mathrm{AB}$ boundaries across which per sample $N$ increases. Both $\mathrm{SHE}_{\text {bip }}$ and $\mathrm{SHE}_{\text {bi }}$ placed a boundary between samples T34 and T3-7 (between ABs $5 \backslash 6$ and $E \backslash F$, resp.). Above this boundary, $\mathrm{SHE}_{\mathrm{bi}}$ grouped the next five samples as $\mathrm{ABF}$. In contrast, $\mathrm{SHE}_{\text {bip }}$ grouped the succeeding two samples T3-7 $(N=67)$ and T3-5 $(N=38)$ as AB4, and then distinguished the succeeding T3-3 $(N=355)$ as a separate AB7. The samples in AB6 contained means of $\sim 25 \%$ T. advena, $\sim 18 \%$ Ammotium distinctum, $\sim 12 \%$ T. inflate, and $12 \%$ Triloculina oblonga, together with 13\%-24\% Ammonia sp. and 0\%-24\% Trochammina inflata. The assemblage in the single sample AB7, in contrast, contained $\sim 60 \%$ T. advena, $18 \%$ T. oblonga and $0 \%$ each of Ammonia sp., T. inflata and A. distinctum. Wright and Hay [30] estimated that a sample size of $N$ $=300$ is needed to ensure with $95 \%$ confidence that all species with an abundance of $>1.0 \%$ have been detected. Given that $N$ in T3-3 exceeds this, it is concluded that the disappearance of Ammonia sp., T. inflata and A. distinctum from $A B 7$ is a statistically significant phenomenon. Simultaneous confidence intervals showed that the difference in the proportional abundances of T. advena in ABs 6 and 7 were statistically significant. There thus occurred a distinct change in the assemblage between $\mathrm{AB} 6$ and $\mathrm{AB} 7$ that warrants the placement of the $\mathrm{AB}$ boundary between them, as given by $\mathrm{SHE}_{\text {bip }}$, even though this was not detected by $\mathrm{SHE}_{\mathrm{bi}}$.

It might be argued that $\mathrm{SHE}_{\text {bip }}$ inserts an $\mathrm{AB}$ boundary wherever there is a large change in per sample $N$. One final example demonstrates that this is not the case. Both $\mathrm{SHE}_{\mathrm{bi}}$ and $\mathrm{SHE}_{\text {bip }}$ place sample T3-9 ( $\sim 11 \%$ of total recovery) within an $\mathrm{AB}$ with the preceding sample, despite that fact that in the underlying sample (T2-5) $N$ was only $28(<1 \%$ of total recovery).

The above examples demonstrate that $\mathrm{SHE}_{\text {bip }}$ is useful where $N$ varies significantly between samples. It must be stressed, however, that $\mathrm{SHE}_{\text {bip }}$ is not intended to replace $\mathrm{SHE}_{\mathrm{bi}}$, but rather to allow the recognition of $\mathrm{AB}$ boundaries under marginal circumstances where sample quality is poor and $\mathrm{SHE}_{\mathrm{bi}}$ cannot function fully. This ability to re-examine poor quality data is surely to be welcomed (just as medical patients with rare diseases welcome any advances made in their treatment despite it being based on studies with small sample sizes). $\mathrm{SHE}_{\text {bip }}$ must not, however, be used indiscriminately and seen as a correction for $\mathrm{SHE}_{\mathrm{bi}}$ to be applied under all circumstances. Whereas the values of $M$, $H_{A}, S_{A}$, and $E_{A}$ from $S_{H E} E_{\text {bi }}$ can be further analysed using $\mathrm{SHE}$ Analysis Identification of Community Structure (SHECSIsee [18]), those from $\mathrm{SHE}_{\text {bip }}$ cannot. 


\section{Conclusions}

If the number of specimens in the samples taken along a line transect varies markedly, $\mathrm{SHE}_{\mathrm{bi}}$ may place an $\mathrm{AB}$ boundary at an unexpected position. In these cases $\mathrm{SHE}_{\mathrm{bi}}$ may be modified by using a table of proportional abundances as a starting point, the new method being termed $\mathrm{SHE}_{\text {bip }}$. Thus, abundance biozone boundaries can now be detected with confidence in situations where specimen recovery from samples is highly variable. Although $\mathrm{SHE}_{\text {bip }}$ was here applied to intertidal foraminifera, it can be applied to any community in which $N$ (per sample) fluctuates markedly. Both $\mathrm{SHE}_{\mathrm{bi}}$ and $\mathrm{SHE}_{\text {bip }}$ can be conducted using spreadsheet programmes that come ready-installed on any new computer. $\mathrm{SHE}_{\text {bip }}$ is especially useful in situations where the number of specimens varies markedly from sample to sample.

\section{Glossary of Symbols}

$N$ : the number of specimens picked from a sample

$n_{i}$ : the number of specimens of the $i$ th species in a sample

$p_{i}: \quad$ the proportional abundance of the $i$ th species in a sample, $n_{i} / N$

$M: \quad$ the number of specimens in an accumulated series of samples

L: $\quad$ the number of samples in an accumulated series of samples

S: $\quad$ the number of species present in a single sample

$\mathrm{S}_{A}$ : the number of species in an accumulated series of samples

$H$ : $\quad$ the value of the information function for a single sample, $H=-\Sigma p_{i} * p_{i}$

$H_{A}$ : the value of the information function for an accumulated series of samples.

$E: \quad$ the value of the equitability index for a single sample, $E=e^{H} / S$

$E_{A}$ : the value of the equitability index for an accumulated series of samples

$\mathrm{SHE}_{\mathrm{bi}}$ : SHE Analysis for Biozone Identification conducted using a matrix of species absolute abundances

$\mathrm{SHE}_{\text {bip }}$ : SHE Analysis for Biozone Identification conducted using a matrix of species proportional abundances.

\section{Acknowledgments}

Thanks are due to bpTT Limited, who financed this paper and to Marty Buzas (Smithsonian Institution, Washington, DC) for comments during this paper's very early stages. A contribution from the Campus Research and Publications Fund of the University of the West Indies, Trinidad, is gratefully acknowledged.

\section{References}

[1] L.-A. C. Hayek and M. A. Buzas, Surveying Natural Populations, Columbia University Press, New York, NY, USA, 1997.

[2] Q. Li and B. McGowran, "Miocene foraminifera from Lakes Entrance Oil Shaft, Gippsland, southeastern Australia," Association of Australasian Palaeontologists Memoirs, vol. 22, pp. 1$142,2000$.

[3] A. E. Magurran, Ecological Diversity and Its Measurement, Chapman and Hall, London, UK, 1988.

[4] A. E. Magurran, Measuring Biological Diversity, Blackwell, Oxford, UK, 2004.

[5] C. E. Shannon, "A mathematical theory of communication," Bell System Technical Journal, vol. 27, pp. 379-423, 623-656, 1948.

[6] C. J. Krebs, Ecological Methodology. Benjamin Cummings, Pearson Education, Upper Saddle River, NJ, USA, 1998.

[7] L. Jost, "Entropy and diversity," Oikos, vol. 113, no. 2, pp. 363375, 2006.

[8] R. Margelef, "Homage to Evelyn Hutchinson, or why is there an upper limit to diversity," Transactions of the Connecticut Academy of Arts and Science, vol. 44, pp. 211-235, 1972.

[9] R. M. May, "Patterns of species abundance and diversity," in Ecology and Evolution of Communities, M. L. Cody and J. M. Diamond, Eds., Harvard University Press, Cambridge, Mass, USA, 1975.

[10] L. Jost, "Partitioning diversity into independent alpha and beta components," Ecology, vol. 88, no. 10, pp. 2427-2439, 2007.

[11] R. H. Whittaker, "Evolution and measurement of species diversity," Taxon, vol. 21, pp. 213-251, 1972.

[12] M. A. Buzas and T. G. Gibson, "Species diversity: Benthonic Foraminifera in Western North Atlantic," Science, vol. 163, no. 3862, pp. 72-75, 1969.

[13] T. G. Gibson and M. A. Buzas, "Species diversity: pattern in modern and Miocene foraminifera of the eastern margin of North America," Geological Society of America Bulletin, vol. 84, pp. 217-238, 1973.

[14] A. L. Sheldon, "Equitability indices: dependence on the species count," Ecology, vol. 50, pp. 466-467, 1969.

[15] M. A. Buzas and L.-A. C. Hayek, "She analysis for biofacies identification," Journal of Foraminiferal Research, vol. 28, no. 3, pp. 233-239, 1998.

[16] R. Sagar, A. S. Raghubanshi, and J. S. Singh, "Asymptotic models of species-area curve for measuring diversity of dry tropical forest tree species," Current Science, vol. 84, no. 12, pp. 1555-1560, 2003.

[17] M. A. Buzas, R. K. Smith, and K. A. Beem, Ecology and Systematics of Foraminifera in Two Thalassia habitats, Jamaica, West Indies, vol. 31 of Smithsonian Contributions to Paleobiolology, Smithsonian Institute, Washington, DC, USA, 1977.

[18] M. A. Buzas, "Community structure of foraminifera from two miocene beds at Calvert Cliffs, Maryland," Journal of Foraminiferal Research, vol. 34, no. 3, pp. 208-213, 2004.

[19] B. Wilson, "Population structures among epiphytal foraminiferal communities, Nevis, West Indies," Journal of Micropalaeontology, vol. 27, no. 1, pp. 63-73, 2008.

[20] R. A. Reyment, Introduction to Quantitative Paleoecology, Elsevier, Amsterdam, The Netherlands, 1971.

[21] G. E. Hutchinson, An Introduction to Population Ecology, Yale University Press, New Haven, Conn, USA, 1978.

[22] L. Jost, "Mismeasuring biological diversity: response to Hoffmann and Hoffmann (2008)," Ecological Economics, vol. 68, pp. 925-928, 2009. 
[23] B. Wilson, "Using SHEBI (SHE analysis for biozone identification): to proceed from the top down or the bottom up? A discussion using two Miocene formaniferal successions from Trinidad, West Indies," Palaios, vol. 23, no. 9-10, pp. 636-644, 2008.

[24] W. Lamboy and A. Lesnikowska, "Some statistical methods useful in the analysis of plant paleoecological data," Palaios, vol. 3, no. 1, pp. 86-94, 1988.

[25] B. Wilson, K. Miller, A.-L. Thomas, N. Cooke, and R. Ramsingh, "Foraminifera in the mangal at the Caroni Swamp, Trinidad: diversity, population structure and relation to sea level," Journal of Foraminiferal Research, vol. 38, no. 2, pp. 127136, 2008.

[26] R. Todd and P. Bronnimann, "Recent foraminifera and thecamoebina from the eastern Gulf of Paria," Cushman Foundation for Foraminiferal Research, vol. 3, pp. 1-43, 1957.

[27] J. B. Saunders, "Trochamminidae and certain Lituolidae (Foraminifera) from the Recent brackish-water sediments of Trinidad, British West Indies," Smithsonian Miscellaneous Collection, vol. 134, pp. 1-16, 1957.

[28] J. B. Saunders, "Recent foraminifera of mangrove swamps and river estuaries and their fossil counterparts in Trinidad," Micropaleontology, vol. 4, pp. 79-92, 1958.

[29] E. Boltovskoy and S. Hincapié de Martínez, "Foraminiferos del Manglar de Tesca, Cartegena, Colombia," Revista Espanola de Micropaleontología, vol. 15, pp. 205-220, 1983.

[30] R. C. Wright and W. W. Hay, "The abundance and distribution of foraminifera in a back-reef environment, Molasses Reef, Florida," Miami Geological Society Memoir, vol. 1, pp. 121-174, 1971. 

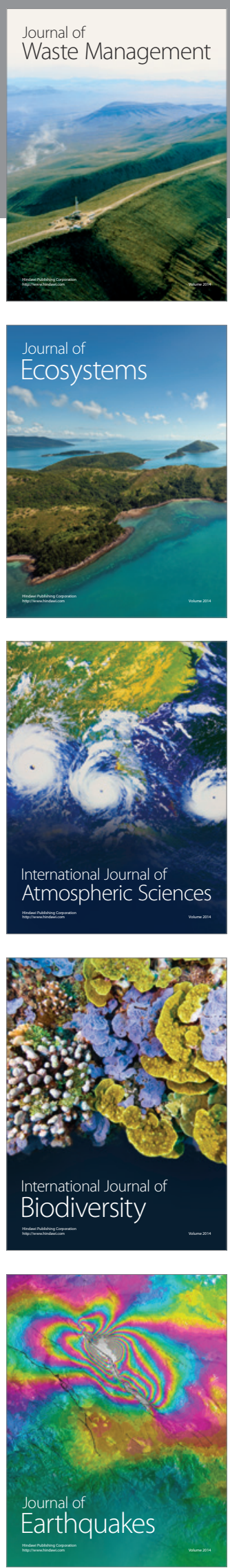
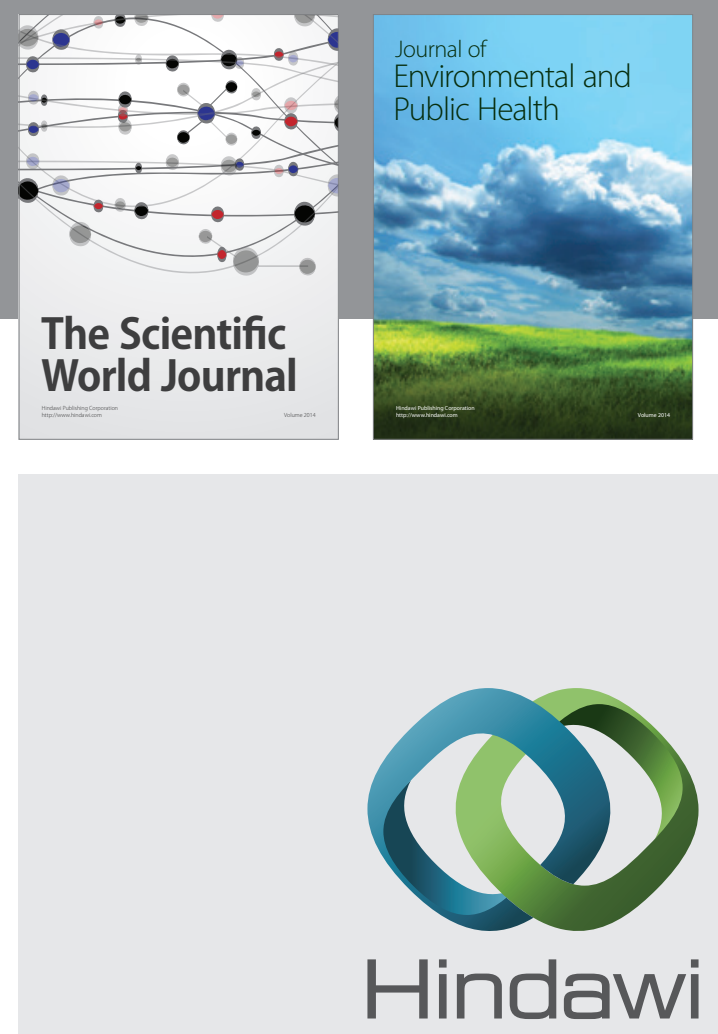

Submit your manuscripts at

http://www.hindawi.com
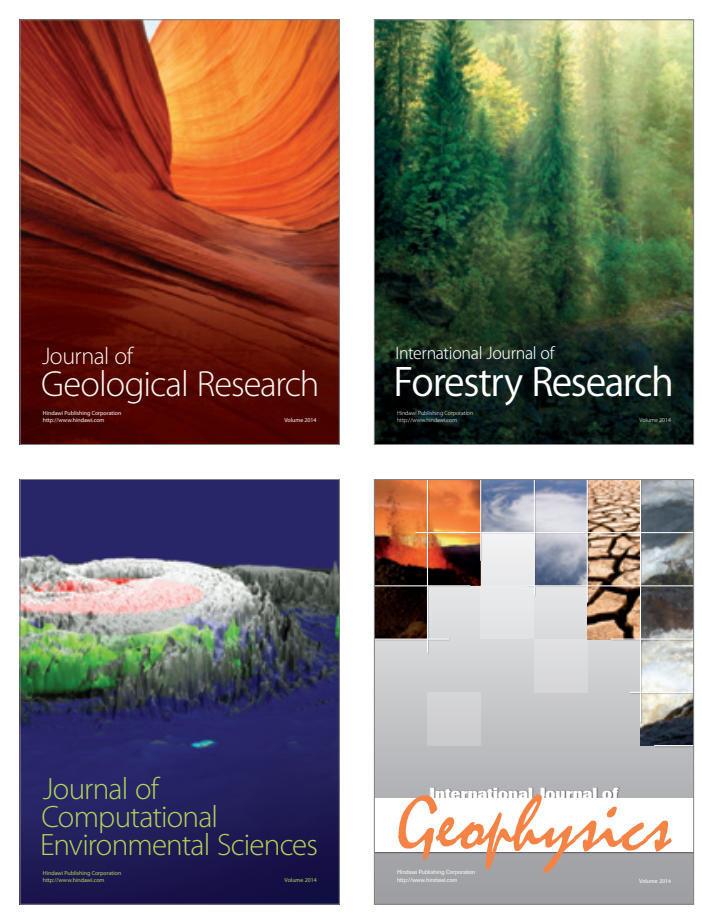
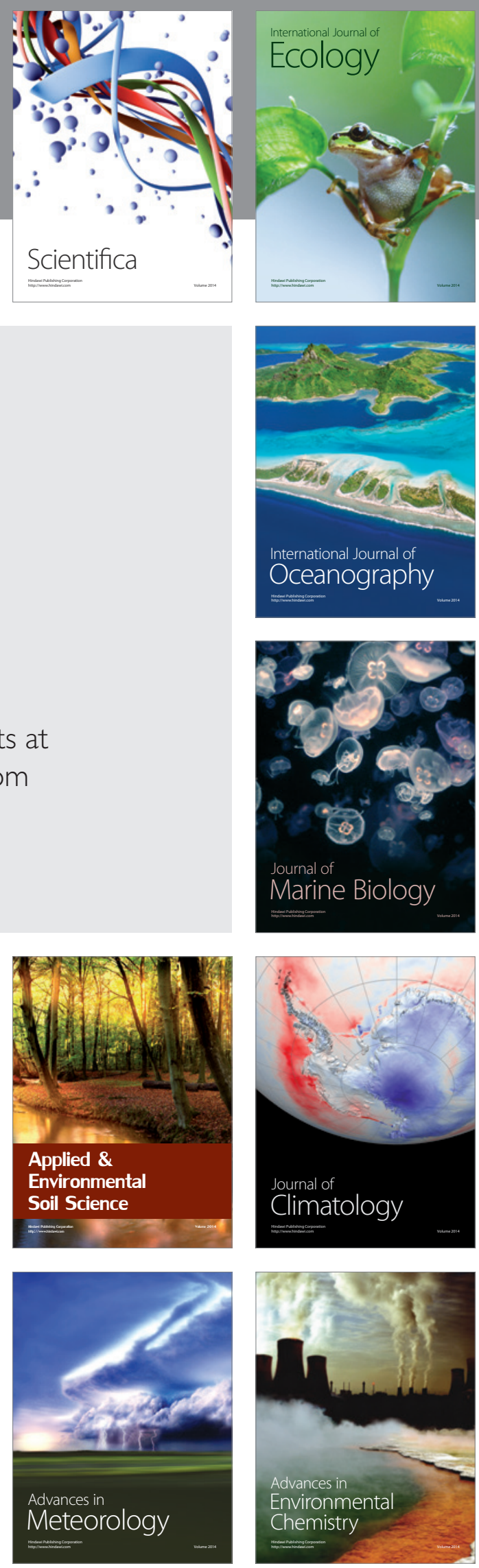